\title{
REVIEWS
}

\section{Emergency oxygen therapy for the breathless patient. Guidelines prepared by North West Oxygen Group}

\author{
R Murphy, K Mackway-Jones, I Sammy, P Driscoll, A Gray, R O’Driscoll, J O'Reilly, \\ R Niven, A Bentley, G Brear, R Kishen
}

Based on a systematic review of the scientific literature, the North West Oxygen Group have developed guidelines for oxygen therapy for patients who present with acute breathlessness. The above emergency medicine physicians, chest physicians and intensive care physicians have gained approval from their regional societies to have this document accepted as the agreed regional guidelines for the use of oxygen in the immediate care of breathless patients in the North West of England. Flow charts are also currently being developed, based on these guidelines, for use by ambulance and emergency department staff in the area.

It is recognised that the present use of oxygen across these specialties is inconsistent. This protocol will help us to deliver standardised oxygen therapy to breathless patients by paramedics, doctors and nurses. This will also improve the consistency of medical training across these disciplines in the North West.

\section{Introduction}

It is crucial to provide optimal oxygen therapy while the acutely breathless patient is being transferred to hospital, assessed in the emergency department and treated for their disease. For most such patients, the main concern is to give sufficient oxygen to support their needs. The major risk is giving too little oxygen (hypoxia). Insufficient oxygen therapy can lead to cardiac arrhythmias, tissue damage, renal damage and, ultimately, cerebral damage. However, excessive oxygen therapy can be dangerous for some patients with respiratory failure.

\section{Problems faced by ambulance staff}

Patients who present acutely with breathlessness will have varying requirements for oxygen therapy depending on the underlying cause of their symptoms. Most acutely breathless patients will have conditions such as asthma, heart failure, pneumonia, pleural effusions, pulmonary embolism or pneumothorax and some may be victims of major trauma. These patients require high concentration oxygen therapy. For most of these patients, $40 \%-60 \%$ oxygen will be sufficient to maintain satisfactory oxygenation (for example, 4-10 1/min from a medium concentration mask depending on the brand-check mask packaging for details) but some patients will require 100\% oxygen from a non-rebreathing reservoir mask. The oxygen delivery system should be adjusted to maintain a saturation greater than $90 \%$.

A proportion of breathless patients will have chronic bronchitis and emphysema. In a survey of 11000 emergency medical admissions to Hope Hospital, Salford between August 1998 and July 1999 it was established that 25\%$30 \%$ had conditions such as COPD (6.2\%), asthma $(5.5 \%)$, pneumonia $(2.9 \%)$, heart failure $(3.8 \%)$ or other respiratory or cardiac diseases that were likely to have presented with acute breathlessness. Based on these figures it is likely e that about a quarter of patients with acute breathlessness will have COPD as their main diagnosis (Dr Ronan O'Driscoll, unpublished observation). Many of these patients will require controlled oxygen therapy because they are at risk of carbon dioxide retention or respiratory acidosis. Data from the study by Plant $e t$ $a l$, referred to in the previous article, indicate that $47 \%$ of COPD patients admitted to their hospital had a $\mathrm{PaCO}_{2}$ above $6.0 \mathrm{kPa}, 20 \%$ had a respiratory acidosis $(\mathrm{pH}<7.35), 4.6 \%$ had a severe acidosis $(\mathrm{pH}<7.25)$ and that acidosis was commoner if the blood oxygen was above $10 \mathrm{kPa}$.

As most ambulance journeys in urban areas in England and Wales will be less than 15 minutes, this risk of hypercapnia, developing or becoming worse as a result of oxygen therapy, should be minimal for most patients if the oxygen saturation is maintained at $90 \%-92 \%$. Rural ambulance journeys may be much longer and hypercapnia may be a risk for some COPD patients. In these circumstances, patients with previous episodes of hypercapnia should be identified and treated with either low flow oxygen from a medium concentration mask (2-4 $1 /$ min equivalent to approximately $28 \%-40 \%$ oxygen) or preferably with controlled oxygen $(24 \%$ or $28 \%$ ) from a Venturi device to maintain an oxygen concentration of $90 \%-92 \%$ during the ambulance journey. It may be appropriate to use "Alert Bracelets" for this group of patients suggesting optimal therapy based on previous blood gas measurements. 
Problems faced by hospital staff

Once transferred to hospital, breathless patients need to be assessed immediately to identify the cause of their breathlessness. For the majority of patients, the main issue is the avoidance of hypoxia but about $12 \%$ of breathless medical patients are at risk of hypercapnic (type II) respiratory failure according to the above figures. In these special circumstances, high concentration oxygen therapy may be harmful. Most such patients have COPD (chronic bronchitis and emphysema) with reduced respiratory drive. However, there are some other circumstances in which type II respiratory failure may occur. Emergency medical teams should consider the following possibilities in patients with a raised $\mathrm{PaCO}_{2}$ level with or without hypoxia:

CAUSES OF TYPE II RESPIRATORY FAILURE

(MANAGEMENT DIFFERS FOR EACH GROUP)

- COPD (especially "blue bloaters" with additional heart failure).

- Cystic fibrosis with severe airway obstruction

- Very severe asthma (needs high dose oxygen and management in ICU, not low dose oxygen therapy)

- Excessive use or overdose of narcotics/ sedatives (These patients will not feel breathless and they require naloxone with high dose oxygen, not low dose oxygen therapy)

- Severe kyphoscoliosis

- Neuromuscular disease affecting respiratory muscles (may require ventilatory support)

- Gross obesity (body mass index above 40 $\mathrm{kg} / \mathrm{m}$. Type II failure common during sleep)

- Extensive previous chest disease (for example, extensive post-tuberculous scarring or lung resection)

To identify such patients, it is important to have a high index of suspicion and to perform arterial blood gas measurements at the earliest opportunity. Reliance on oxygen saturation alone in these patients may be misleading. Each of these patient groups may require very different medical management.

OTHER PRINCIPLES OF OXYGEN THERAPY

- Oxygen therapy should continue during other treatments such as nebulised therapy.

- Some patients, especially those with high respiratory rates, can be made more comfortable (less breathless) by increasing the flow of oxygen without increasing the concentration. This applies especially to low concentration Venturi masks where the gas flow available to the patient at the lowest recommended oxygen flow rate may be much lower than the patient's inspiratory flow rate. These masks provide a fixed oxygen concentration whatever the flow rate provided.

- For breathless patients requiring high concentration oxygen, a well fitted nonrebreathing reservoir mask will increase the amount of oxygen available during inspiration.

- Emergency oxygen treatment is best given by face masks. Nasal prongs are best suited to the treatment of patients who are stable where the flow rate can be titrated to the desired response (based on blood gas analysis). The only role for nasal prongs in emergency treatment is to give supplementary low flow oxygen therapy to patients with known hypercapnic COPD during bronchodilator treatment with air driven nebuliser systems.

- The use of a structured oxygen prescription document has been shown to improve oxygen prescribing and administration in hospitals.

Emergency oxygen therapy for the breathless patient. Guidelines prepared by North West Oxygen Group

\section{The guidelines are divided into three}

stages:

(1) Pre-hospital stage;

(2) Emergency department assessment stage;

(3) Pre-admission stage.

(1) PRE-HOSPITAL STAGE (ACUTELY BREATHLESS PATIENTS)

- In this situation the diagnosis is often unclear and the risk of hypoxia is much greater than the risk of hypercapnia for most patients.

- The main issue for paramedics is to maintain oxygenation.

- These patients should be given high concentration oxygen to maintain an oxygen saturation above $90 \%$ until arrival at an emergency department. This can be achieved in most cases by the use of approximately $40 \%-60 \%$ oxygen via a medium concentration mask. (Oxygen flow of 4-10 1/min depending on brand of mask). Use a reservoir (non-rebreathing) mask if the patient is severely hypoxic and in all major trauma cases.

- For patients with known COPD, it is not desirable to exceed an oxygen saturation of $93 \%$. In these cases, oxygen therapy should be started at approximately $40 \%(4-61 / \mathrm{min}$ for most brands of medium concentration mask) and titrated upwards if the oxygen saturation decreases below $90 \%$ and downwards if the patient becomes drowsy and the saturation exceeds $93-94 \%$.

- For the vast majority of patients in urban areas in the United Kingdom, the journey to hospital will take less than 15 minutes and the risks of hypercapnia are minimal during this short journey. Patients with known type II respiratory failure need special care, especially if they require a prolonged rural ambulance journey (see introductory notes).

(2) EMERGENCY DEPARTMENT ASSESSMENT STAGE (Management of acutely breathless patients before blood gas results become available)

- On arrival at the emergency department, breathless patients with a significant likelihood of severe COPD should be triaged as very urgent (Orange Status). These patients should be seen by a doctor within 10 minutes of arrival in the department. 
- Continuous oximetry should be initiated and blood gases should be measured. The inspired oxygen concentration at the time of blood gas sampling should be noted and recorded. Blood gas measurements need to be repeated after changes in oxygen therapy.

- Although history taking and clinical examination may clarify the diagnosis, oxygen at $40 \%-60 \%$ should be continued until blood gas results are available unless the patient is drowsy or is known to have had previous episodes of hypercapnic respiratory failure. In these circumstances, a lower $\mathrm{FiO}_{2}$ may be required such as $2-41 / \mathrm{min}$ via a medium concentration mask (equivalent to approximately $28 \%-40 \%$ oxygen) or preferably by the use of controlled oxygen at $24 \%$ or $28 \%$ via a Venturi mask titrated upwards or downwards to maintain an oxygen saturation of $90 \%-92 \%$ pending the results of blood gas estimations.

- Oxygen treatment should be given continuously and concurrently with nebulised bronchodilators, if these are indicated, by running the nebuliser on high concentration oxygen (prior to availability of blood gas results).

3 (A) PRE-ADMISSION STAGE AND EARLY

ADMISSION STAGE (NON-COPD PATIENTS)

- Patients with asthma, left ventricular failure, pneumonia, pneumothorax, trauma, etc, should be treated appropriately for their condition using $40 \%-60 \%$ oxygen via a medium concentration mask (4-10 $1 / \mathrm{min})$ for milder cases or a reservoir mask for hypoxic patients and for all major trauma cases.

3 (B) PRE-ADMISSION STAGE AND EARLY

ADMISSION STAGE (COPD PATIENTS)

- In the normocapnic COPD patient, oxygen should be adjusted (by downward titration) to the lowest concentration required to maintain an oxygen saturation of $90 \%-92 \%$. There is no known value in maintaining an oxygen saturation above $93 \%$ in patients with COPD but this may cause respiratory acidosis or worsen pre-existing acidosis.

- If the $\mathrm{PaCO}_{2}$ is raised the history, examination and relevant investigations should be reviewed to confirm that this patient is suffering from an acute exacerbation of COPD (see introductory notes for a list of other causes of type 2 respiratory failure). If a diagnosis of an exacerbation of COPD is confirmed, standard COPD therapy should be given as recommended in the British Thoracic Society COPD Guidelines. Controlled oxygen therapy is best given via a fixed performance Venturi mask at the lowest oxygen concentration required to maintain an oxygen saturation of $90 \%-92 \%$ and satisfactory blood gas levels and $\mathrm{pH}$ level.
When nebulised bronchodilators are given to hypercapnic acidotic patients, they should be driven by compressed air and, if necessary, supplementary oxygen should be given concurrently by nasal prongs at $1-4$ litres per minute to maintain an oxygen saturation of $90 \%-92 \%$. Once the nebulised treatment is completed, controlled oxygen therapy with a Venturi mask should be re-instituted. The $\mathrm{PaCO}_{2}$ and $\mathrm{pH}$ should be monitored every hour or more frequently if there is a clinical deterioration. If the $\mathrm{pH}$ does not decrease below 7.26 and the $\mathrm{PaCO}_{2}$ does not rise above $80 \mathrm{~mm} \mathrm{Hg}(10.6$ $\mathrm{kPa}$ ), and if the patient's condition is considered satisfactory, oxygen therapy at this concentration can be continued. If the $\mathrm{pH}$ falls below 7.26 or the $\mathrm{PaCO}_{2}$ rises above $80 \mathrm{~mm} \mathrm{Hg}$ $(10.6 \mathrm{kPa})$, or if the patient becomes drowsy or fatigued, non-invasive ventilation should be initiated if available. Local guidelines for earlier institution of non-invasive ventilation may apply-recent research has shown benefit from non-invasive ventilation at a $\mathrm{pH}$ from 7.25 to 7.35. Senior advice should be sought and a decision should be made about the appropriateness of invasive ventilation. Doxapram may be considered if non-invasive ventilation is unavailable.

For patients with a rising $\mathrm{PaCO}_{2}$ and a falling $\mathrm{pH}$ in whom mechanical ventilation is not considered appropriate by the consultant physician or emergency department consultant or ICU consultant, the concentration of inspired oxygen could be decreased further as long as the $\mathrm{PaO}_{2}$ does not decrease below $50 \mathrm{~mm} \mathrm{Hg}$ $(6.6 \mathrm{kPa})$ (approximately $80 \%$ oxygen saturation). This may result in a decrease in $\mathrm{PaCO}_{2}$ and a rise in $\mathrm{pH}$. It is recognised that some of these patients may have resting oxygen saturations below $90 \%$ when stable. Again, the patient must be monitored regularly (oximetry and consciousness level) and have regular arterial blood gas analysis until stable.

North West Oxygen Group

Department of Emergency Medicine, Manchester Royal Infirmary: Ross Murphy, Kevin Mackway-Jones; Department of Emergency Medicine, Hope Hospital, Salford: Ian Sammy, Pete Driscoll; Department of Emergency Medicine, Stepping Hill Driscoll; Department of Emergency Medicine, Stepping Hill
Hospital, Stockport: Alistair Gray; Department of Chest Medicine, Hope Hospital, Salford: Ronan O'Driscoll; Department of Chest Medicine, Victoria Hospital, Blackpool: John O'Reilly; Department of Chest Medicine, Wythenshawe Hospital, Manchester: Rob Niven; Department of Intensive Care Medicine, North Manchester General Hospital: Andrew Bentley; Department of Intensive Care Medicine, Wythenshawe Care Medicine, Hope Hospital, Salford: Roop Kishen.

Funding: we are grateful to Boehringer Ingelheim for an educational grant to support a guidelines development meeting and printing costs to assist with the implementation of the guidelines in the North West.

Conflicts of interest: none.

Contributors

Ross Murphy, Peter Driscoll, Alistair Gray and Ronan O'Driscoll initiated the project. All authors, particularly Ronan O'Driscoll, contributed to the development and implementation of the guidelines. Ronan O'Driscoll acts as guarantor for the paper. 